\title{
Effects of husbandry systems and Chinese indigenous chicken strain on cecum microbial diversity
}

\author{
Xiuxue Dong ${ }^{1, \mathrm{a}}$, Bing $\mathrm{Hu}^{1, \mathrm{a}}$, Wenlong Wan ${ }^{1}$, Yanzhang Gong ${ }^{1, *}$, and Yanping Feng ${ }^{1, *}$
}

\author{
* Corresponding Authors: \\ Yanzhang Gong \\ Tel: +86-27-15347112816, Fax: +86-27-87280408, \\ E-mail: poultry@mail.hzau.edu.cn \\ Yanping Feng \\ Tel: +86-27-15347112816, \\ E-mail: fengyanping594@mail.hzau.edu.cn
}

'College of Animal Science and Veterinary Medicine, Huazhong Agricultural University, Wuhan, Hubei 430070, China

a These two authors contributed equally to this work.

ORCID

Xiuxue Dong

https://orcid.org/0000-0002-4177-0357

Bing $\mathrm{Hu}$

https://orcid.org/0000-0001-8463-6919

Wenlong Wan

https://orcid.org/0000-0002-4553-2946

Yanzhang Gong

https://orcid.org/0000-0001-8308-3005

Yanping Feng

https://orcid.org/0000-0003-4723-5408

Submitted Feb 26, 2019; Revised May 8, 2019; Accepted Oct 10, 2019
Objective: This study was to evaluate the effect of husbandry systems and strains on cecum microbial diversity of Jingyang chickens under the same dietary conditions.

Methods: A total of 320 laying hens (body weight, $1.70 \pm 0.15 \mathrm{~kg}$; 47 weeks old) were randomly allocated to one of the four treatments: i) Silver-feathered hens in enrichment cages (SEC) with an individual cage $(70 \times 60 \times 75 \mathrm{~cm})$, ii) Silver-feathered hens in free range (SFR) with the stocking density of 1.5 chickens per ten square meters, iii) Gold-feathered hens in enrichment cages (GEC), iv) Gold-feathered hens in free range (GFR). The experiment lasted 8 weeks and the cecum fecal samples were collected for $16 \mathrm{~S}$ rDNA high throughput sequencing at the end of experiment.

Results: i) The core microbiota was composed of Bacteroidetes (49\% to 60\%), Firmicutes (21\% to $32 \%)$ and Proteobacteria (2\% to $4 \%$ ) at the phylum level. ii) The core bacteria were Bacteroides ( $26 \%$ to $31 \%$ ), Rikenellaceae ( $9 \%$ to $16 \%$ ), Parabacteroides ( $2 \%$ to $5 \%$ ) and Lachnoclostridium (2\% to 6\%) at the genus level. iii) The indexes of operational taxonomic unit, Shannon, Simpson and observed species were all higher in SFR group than in SEC group while in GEC group than in GFR group, with SFR group showing the greatest diversity of cecum microorganisms among the four groups. iv) The clustering result was consistent with the strain classification, with a similar composition of cecum bacteria in the two strains of laying hens.

Conclusion: The core microbiota were not altered by husbandry systems or strains. The free-range system increased the diversity of cecal microbes only for silver feathered hens. However, the cecum microbial composition was similar in two strain treatments under the same dietary conditions.

Keywords: Jingyang Chicken; Husbandry System; Cecum Microbiome; Diversity; Illumina MiSeq Sequencing

\section{INTRODUCTION}

Asian indigenous chickens under the traditional husbandry system (free range) enjoy the advantages of better adaptation to endemic diseases and other harsh environmental stresses as well as requiring minimal care and low cost, which are in accordance with the wishes of local farmers [1]. The meat and eggs of some indigenous chickens are preferred over those of exotic breeds because of their unique flavor; meanwhile, the international voice for animal welfare allows the chicken industry to become more competitive by stressing the welfare advantages of the free-range system. This system is not only environmentally friendly and enables the animals to enjoy higher welfare standards and produce agricultural products with better quality and better flavor [2], but also provides job opportunities to the low-income population in rural areas [3].

Jingyang chicken, a Chinese indigenous breed from Jianshi county, Hubei, China with 
a unique appearance and good meat quality, has two strains of silver feather and gold feather. The quality and flavor of chicken products are related to the intestinal microbial community, which not only provides nutrition for the host, but also helps to maintain the balance of the intestinal microecology, and ensures the health and development of the intestinal tract [4, 5]. Particularly, the core flora of intestinal tract directly affects its normal function [6], which is closely related to the chicken products such as meat and eggs. Moreover, intestinal microbial community can be jointly affected by genetics and husbandry systems. The gut microflora is a complex ecosystem with a symbiotic relationship to the host, and interactions between them affect the physiological, immune and nutritional status of the host [7]. In general, the intestinal microbial system mainly focuses on microbial community composition and diversity. The intestinal microbial diversity is essential for animals to digest and absorb nutrients, maintain intestinal biochemical and physiological functions, and promote the development of immune system $[8,9]$.

Unlike ruminants which mainly depend on ruminal microorganisms to digest cellulose, monogastric animals such as chickens are primarily dependent on the fermentation and degradation of microbes in the large intestine for digestion of cellulose [10]. Besides genetic factors, husbandry systems also make important contributions to the diversity of cecum microflora. Compared with caged chickens, free-range chickens have free access to feed herbage, seeds and insects, leading to accumulation of many intestinal microbes from the environment and formation of cecum microbial communities. Therefore, studies on the formation and diversity (species and richness) of the cecum microflora are of great significance for improving animal production performance and product quality.

In recent years, cecum microorganism has been a research focus, and a range of techniques have been developed to facilitate its research, such as denaturing gradient gel electrophoresis [11], terminal restriction fragment length polymorphism (T-RFLP) [12], real-time quantitative reverse transcriptionpolymerase chain reaction (qRT-PCR) [13] and anaerobic cultivation [14]. In this study, Illumina MiSeq sequencing was adopted due to its advantages of high resolution for characterization of the microbial community, large coverage and low cost [15].

Diet has been reported as the biggest determinant of the composition of chicken intestinal flora [16]. The primary aim of this study was to explore the effects of the two variables (husbandry system and strain) on the chicken cecum microorganisms under the same dietary conditions. The results of this study can provide theoretical guidance to the farmers who are engaged in ecological breeding of indigenous chickens.

\section{MATERIALS AND METHODS}

\begin{abstract}
Animal care
Animal experiments were performed under the institutional guidelines of Ecological Chicken Farm in Jianshi County of Hubei Province and Laboratory of Poultry Genetics, College of Animal Science and Veterinary Medicine, Huazhong Agricultural University, China. The experiments were performed according to recommendations proposed by the European Commission (1997) to minimize the suffering of animals.
\end{abstract}

\section{Experimental design and administration}

Jingyang chickens were reared in the Fire Phoenix Ecological Breeding Cooperative of Jianshi County (Hubei, China). A total of 320 laying hens (body weight, $1.70 \pm 0.15 \mathrm{~kg}$; 47 weeks old), derived from silver feather (S-line $=160$ hens) and gold feather (G-line $=160$ hens), under the two husbandry systems of enrichment cages (EC) and free range (FR) were randomly divided into four groups (80 hens per group): silver-feathered hens in enrichment cages (SEC), silver-feathered hens in free range (SFR), gold-feathered hens in enrichment cages (GEC), and gold-feathered hens in free range (GFR). For EC husbandry system, each hen was reared in an individual cage $\left(70 \times 60 \times 75 \mathrm{~cm}\right.$ ) with controlled conditions of $18^{\circ} \mathrm{C}$ to $22^{\circ} \mathrm{C}$, $16 \mathrm{~L} / 8 \mathrm{D}$ photoperiod/day. For FR husbandry system, chickens were raised in mountain areas characterized by arbor evergreen coniferous in the visible small stones and grains of sand, with a stocking density of 1.5 chickens per 10 square meters. Chickens in EC and FR were fed the same local standard diet (2,700 kcal $/ \mathrm{kg}, 13 \%$ protein, $1 \%$ calcium, $0.45 \%$ phosphorous) (DB 42/T 686-2011) and drinking water ad libitum during the experiment period.

\section{Sampling and measurements}

At the end of the 60-day experiment, 5 chickens were randomly selected from each treatment (a total of 4 treatments $\times 5$ repeat $=20)$ and the cecal samples were collected in a cryopreservation tubes and then mixed and stored in liquid nitrogen at $-70^{\circ} \mathrm{C}$.

Microbial genome DNA was extracted from cecal samples by using QIAamp DNA stool mini kit (Tiangen Biotech, Beijing, China) according to the manufacturer's instructions. PCR amplification of the V3-V4 region of the16S ribosomal RNA (rRNA) gene was performed using the $341 \mathrm{~F} / 802 \mathrm{R}$ primer set (341F: CCTAYGGGRBGCASCAG; 802R: GGACTAC NNGGGTATCTAAT) as previously reported [17]. Only the products without primer dimers and contaminant bands were used for $16 \mathrm{~S}$ rDNA high throughput sequencing at Shanghai Personal Biotechnology Co., Ltd (Shanghai, China).

\section{Data analysis}

The screened and obtained high-quality sequences uploaded 
to quantitative insights into microbial ecology (QIIME, v1.8.0), were clustered into operational taxonomic units (OTUs) with a sequence similarity of $>97 \%$ using the USEARCH software.

\section{RESULTS AND DISCUSSION}

\section{Operational taxonomic unit analysis results of cecum microbes}

The OTU analysis results of cecum microbes are presented in Table 1 and 2. A total of 22,660 OTUs consisted of 5,880, 6,245, 6,316, and 4,219 OTUs in SEC, GEC, SFR, and GFR, respectively (Table 1), and the annotation results are shown at various classification levels and the number of annotations in species is far less than that in genus level (Table 2). Moreover, the rarefaction curve (Figure 1) tends to be flat as sequencing depth increases and the number of OTUs reaches a plateau, with a sample coverage rate of over 0.96 (Table 3 ). These results confirmed that our sequencing has covered most of the sample species, and the sequencing results fully reflect the microbial diversity of the samples.

\section{Abundance and diversity analysis of cecum microbiota} The results of abundance and diversity analysis are presented in Table 3. In silver-feathered chickens, Shannon index and Simpson index of SFR were higher than those of SEC. Nevertheless, compared with GFR, GEC showed higher values of chaol, Shannon index, and Simpson index. Bailey et al [18] found that exposure to prolonged restraint pressure (a stress method used to induce physiological responses in an animal by restricting its free movement) results in a decrease in the abundance and diversity of intestinal flora in mice. Thus, some of the above results in the present study could be explained by a prolonged restraint pressure-induced decrease in the relative abundance of bacteria of chickens in cages. However, Chaol, a species richness index, was lower in free-range groups than in cage groups. In our former research, a higher level of lactobacilli was observed in free range group, which could inhibit the proliferation of other bacteria and promote gut health [19]. GFR showed the maximum content of Lactobacillus, but the minimum content of chaol, Shannon index, and Simpson index in our present study. These data demonstrated that Lactobacillus did inhibit the growth of some bacteria.

\section{Microbial community composition in cecum}

The core microbiota of the 4 groups were counted at the phylum level (Table 4) and genus level (Table 5). At the phylum level, core members are microbes belonging to Bacteroidetes ( $49 \%$ to $60 \%$ ), Firmicutes (21\% to $32 \%$ ), and Proteobacteria ( $2 \%$ to $4 \%$ ), which are similar to the results reported in broilers [20], Dagu chickens [21], and Tibetan Chickens [22]. It is reported that the total proportion of Firmicutes and Bacteroides is even more than $90 \%$ in some mammalian species $[23,24]$, and the Firmicutes/Bacteroides ratio can directly reflect the capacity of energy absorption and storage of host, such as the increase of the proportion of Firmicutes due to the deposition of fat [25], or the increase of the proportion of Bacteroides with the decrease of the Firmicutes/Bacteroides

Table 1. Sequencing data and OTUs annotation

\begin{tabular}{lcccccc}
\hline Group $^{1)}$ & Raw PE & Raw tags $^{2)}$ & Clean tags $^{2)}$ & Effective tags $^{2)}$ & Avg Len $^{2)}$ & OTUs number $^{2)}$ \\
\hline SEC & 100,563 & 86,795 & 83,500 & 81,123 & 456 & 5,880 \\
SFR & 102,343 & 89,821 & 86,752 & 83,586 & 455 & 6,316 \\
GEC & 92,222 & 79,597 & 76,626 & 73,960 & 457 & 6,245 \\
GFR & 86,700 & 75,441 & 72,728 & 72,027 & 457 & 4,219 \\
\hline
\end{tabular}

OTU, operational taxonomic units.

1) SEC, silver-feathered hens in enrichment cages; SFR, silver-feathered hens in free range; GEC, gold-feathered hens in enrichment cages; GFR, gold-feathered hens in free range.

${ }^{2)}$ Raw PE, the original PE (paired-end) reads; Raw tags, tag sequences obtained after splicing; Clean tags, a sequence with low-quality and short-length tags being removed; Effective tags, tag sequence collected for further analysis after filtering the chimera; Avg Len, the average length of effective tags; OTUs number, the number of OTUs obtained per sample.

Table 2. Classification of OTUs annotation results

\begin{tabular}{lcccccccc}
\hline Group $^{1)}$ & Unassigned & Kingdom & Phylum & Class & Order & Family & Genus & Species \\
\hline SEC & 1,353 & 1 & 20 & 5 & 92 & 719 & 3,673 & 17 \\
SFR & 1,332 & 4 & 19 & 4 & 87 & 733 & 4,119 & 18 \\
GEC & 1,443 & 2 & 17 & 4 & 101 & 721 & 3,944 & 13 \\
GFR & 727 & 2 & 12 & 4 & 71 & 541 & 2,855 & 7 \\
\hline
\end{tabular}

OTU, operational taxonomic units.

1) SEC, silver-feathered hens in enrichment cages; SFR, silver-feathered hens in free range; GEC, gold-feathered hens in enrichment cages; GFR, gold-feathered hens in free range. 


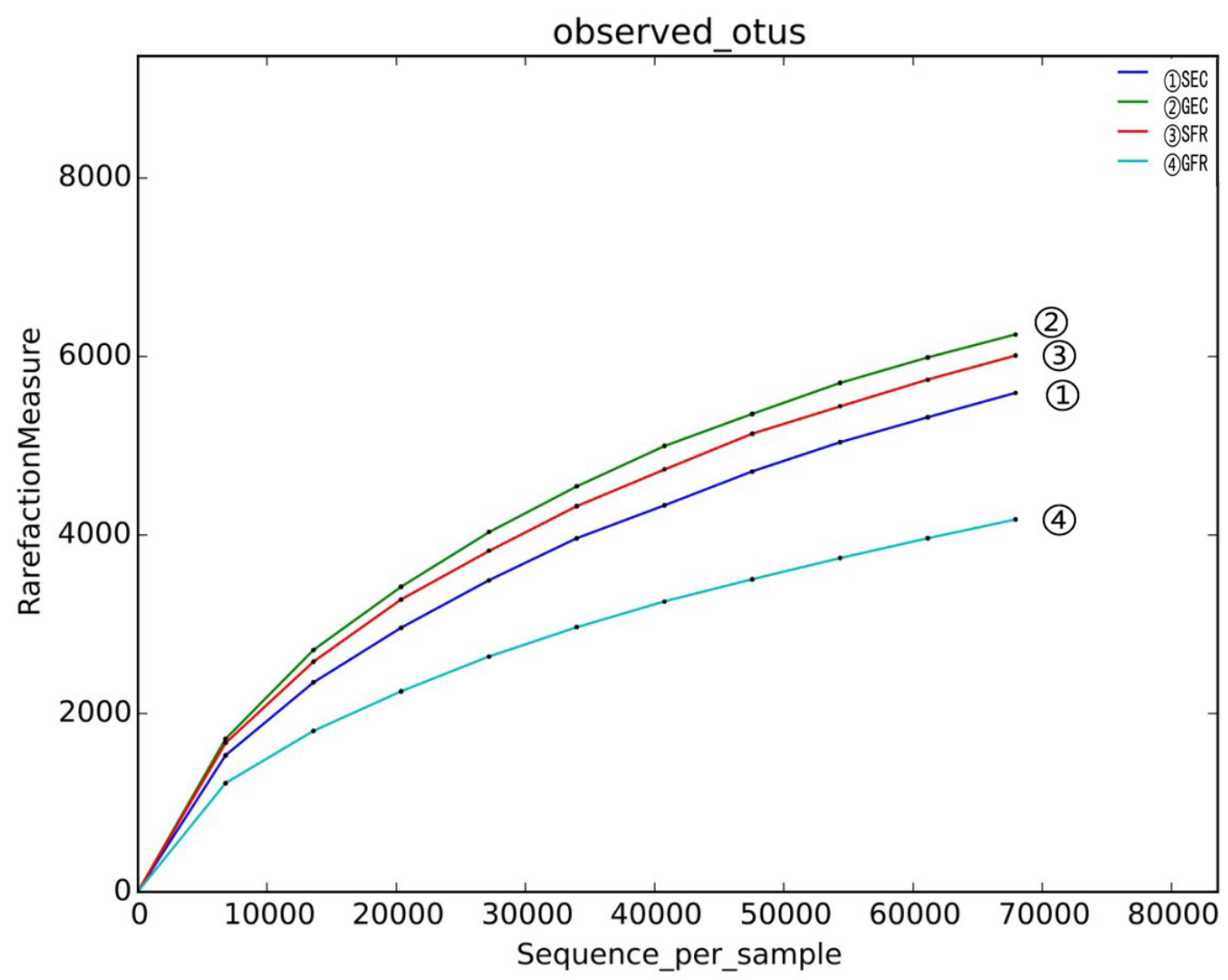

Figure 1. Species rarefaction curve. There is no intersection point after the zero point of the curve, and the order from high to low is GEC, SFR, SEC, GFR respectively.

Table 3. Abundance and diversity analysis of cecum microbiota

\begin{tabular}{lccccc}
\hline Group $^{1)}$ & Chao1 & Shannon & Simpson & $\begin{array}{c}\text { observed } \\
\text { species }\end{array}$ & $\begin{array}{c}\text { Goods } \\
\text { coverage }\end{array}$ \\
\hline SEC & 8367.58 & 9.05 & 0.99 & 5591.1 & 0.96 \\
SFR & 8218.05 & 9.32 & 0.991 & 6009.3 & 0.96 \\
GEC & 8028.44 & 9.13 & 0.989 & 6244.9 & 0.97 \\
GFR & 6752.82 & 8.44 & 0.987 & 4173.7 & 0.97 \\
\hline
\end{tabular}

1) SEC, silver-feathered hens in enrichment cages; SFR, silver-feathered hens in free range; GEC, gold-feathered hens in enrichment cages; GFR, gold-feathered hens in free range.

ratio due to enormous absorption of cellulose food $[23,26]$. In our study, the total proportion of Bacteroidetes and Firmicutes was more than $75 \%$ in Jingyang chickens, which was higher in free-range groups (SFR 81.61\%; GFR 82.12\%) than in enrichment-cage groups (SEC 76.38\%; GEC 75.33\%). The ratio of Firmicutes/Bacteroides was lower in GFR (23.00\%/ $59.12 \%)$ than in GEC (21.20\%/54.13\%), whereas opposite results were obtained for silver-feather hens (SFR vs SEC). The reduced ratio of Firmicutes/Bacteroides in GFR group may be attributed to the higher cellulose food intakes in free range environment.

The core bacteria were Bacteroides (26\% to 31\%), Rike-
Table 4. Phylum distribution of cecum bacteria

\begin{tabular}{lcccc}
\hline Items & $\mathbf{S E C}^{\mathbf{1}}$ & SFR $^{1)}$ & GEC $^{1)}$ & GFR $^{1)}$ \\
\hline Bacteroidetes & 50.12 & 49.66 & 54.13 & 59.12 \\
Firmicutes & 26.26 & 31.95 & 21.2 & 23 \\
Proteobacteria & 3.26 & 2.87 & 3.11 & 2.35 \\
Spirochaetae & 0.82 & 0.78 & 2.21 & 1.04 \\
Synergistetes & 1.31 & 0.59 & 0.78 & 0.23 \\
Saccharibacteria & 0.3 & 0.38 & 0.26 & 0.71 \\
SHA-109 & 0.54 & 0.6 & 0.17 & 0.23 \\
Actinobacteria & 0.53 & 0.6 & 0.21 & 0.59 \\
Verrucomicrobia & 0.21 & 0.29 & 0.05 & 0.12 \\
Euryarchaeota & 0.15 & 0.12 & 0.09 & 0.21 \\
Others & 16.51 & 12.15 & 17.81 & 12.39
\end{tabular}

1) SEC, silver-feathered hens in enrichment cages; SFR, silver-feathered hens in free range; GEC, gold-feathered hens in enrichment cages; GFR, gold-feathered hens in free range.

nellaceae ( $9 \%$ to $16 \%$ ), Parabacteroides ( $2 \%$ to $5 \%$ ), and Lachnoclostridium (2\% to $6 \%$ ) at the genus level. Ruminococcaceae was higher in SFR (2.25\%) than in SEC $(1.23 \%)$ as well as higher in GFR (1.4\%) than in GEC (0.78\%). Combined with our previous research results on cecum index, we speculate that the increased of free-range husbandry in 
Table 5. Genus distribution of cecum bacteria

\begin{tabular}{lcccc}
\hline Items & SEC $^{1)}$ & SFR $^{1)}$ & GEC $^{\text {1) }}$ & GFR $^{1)}$ \\
\hline Bacteroides & 27.87 & 26.42 & 28.86 & 30.55 \\
Rikenellaceae & 9.03 & 11.73 & 11.29 & 15.31 \\
Parabacteroides & 4.75 & 3.45 & 2.24 & 2.52 \\
Lachnoclostridium & 3.37 & 5.1 & 2.75 & 3.51 \\
Desulfovibrio & 2.32 & 2.21 & 2.56 & 1.89 \\
Prevotellaceae & 2.35 & 1.61 & 4.03 & 3.15 \\
Ruminococcaceae & 1.23 & 2.25 & 0.78 & 1.4 \\
Christensenellaceae & 1.11 & 1.52 & 0.6 & 0.8 \\
Others & 47.98 & 45.71 & 46.89 & 40.86 \\
\hline
\end{tabular}

1) SEC, silver-feathered hens in enrichment cages; SFR, silver-feathered hens in free range; GEC, gold-feathered hens in enrichment cages; GFR, gold-feathered hens in free range. the accumulation of microorganisms is related to cellulose degradation, which can be supported by the studies of Saengkerdsub et al [27], Lu et al [28], and Latham et al [29], who reported that ruminococcaceae was responsible for cellulose digestion.

\section{Abundance and clustering analysis of relative bacterial communities}

A further analysis was conducted to discover the relative bacterial community abundance at the phylum level, and the results are shown in Figure 2. The phyla were divided into four groups. Group 1 (Synergistes, Phascolarctobacterium, Megasphaera, Megamonas, Sutterella, and Parabacteroides) was predominant in SEC. Group 2 (Ruminococcaceae, Lachnoclostridium, Alloprevotella, Christensenellaceae, Oribacterium,

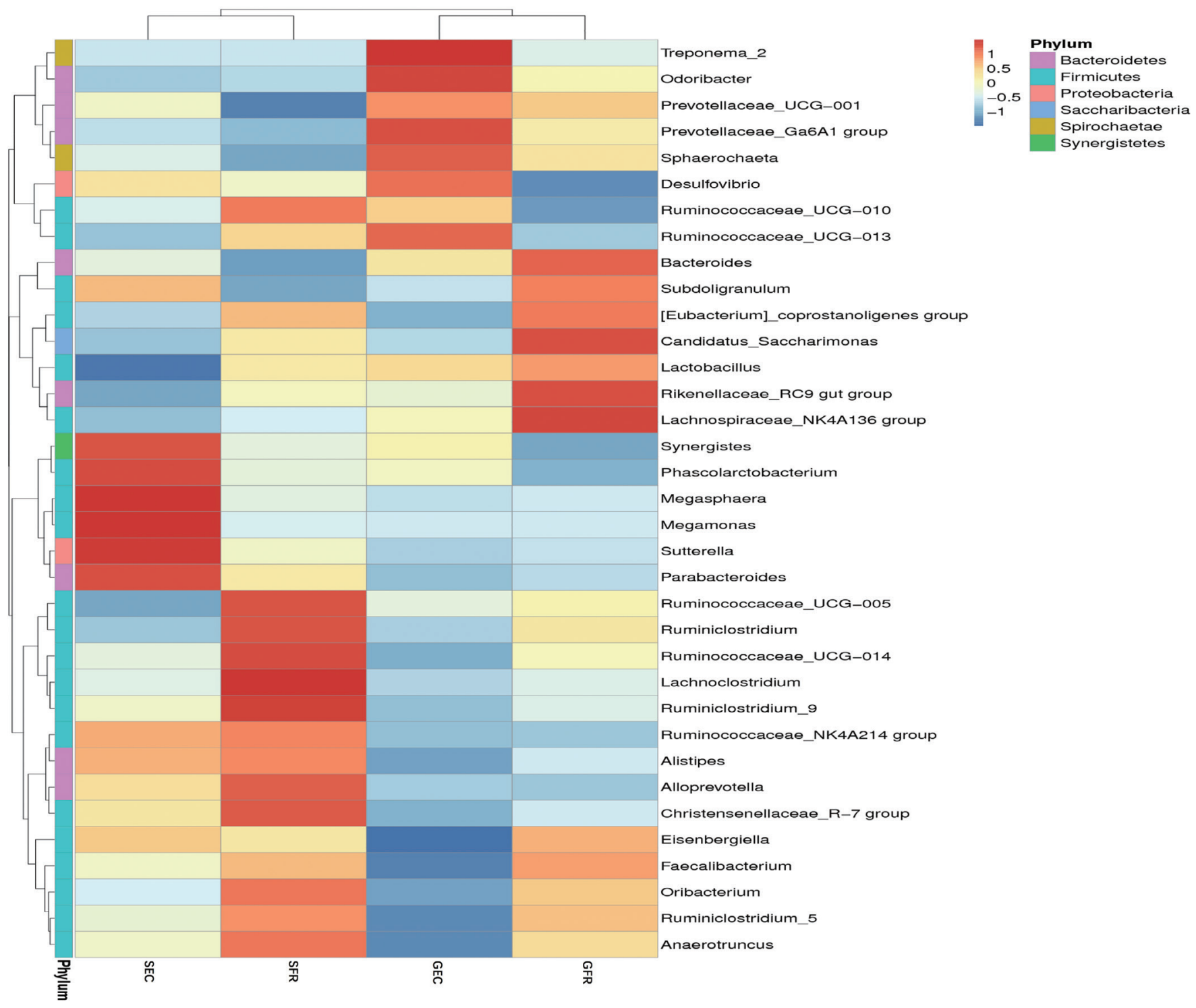

Figure 2. Heat maps of species abundance and clustering. The phylum classification position clustering (horizontal) and top 35 phylum sample clustering (vertical clustering). Different color means the different relative abundance of the phylum in all the four samples. 
and Anaerotruncus) was typical in SFR. Group 3 (Treponema, Odoribacter, Prevotellaceae, Sphaerochaeta, Desulfovibrio, and Ruminococceae) and Group 4 (Bacteroides, Subdoligranulum, Eubacterium, Saccharimonas, Rikenellaceae, and Lachnoclostridium) were observed in GEC and GFR, respectively. The clustering was dependent on hen strains (gold vs silver).

The clustering result was consistent with the strain classification: the silver-feather strain and the gold-feather strain. Actually, factors affecting the intestinal microbiotas are host and environment, with the former mainly referring to heredity, age and endocrinology, and the latter involving diet, geographical environment, lifestyle, and the use of antibiotics $[16,30]$. Diet has been reported as the dominant factor for the intestinal microbial community in the formation of host genotype [16]. In this study, we explored other factors affecting cecum microbial diversity under the same dietary conditions. Our study proved that the genetic factor has a greater impact than husbandry system on cecum microbial diversity in Jingyang chickens.

\section{CONCLUSION}

In conclusion, Bacteroides is the most abundant in cecum microbiotas of Jingyang chickens, followed by Firmicutes and Proteobacteria. Free range husbandry system as well as strain have observable effects on diversity of the cecum microorganism of Jingyang chickens. However, genetic factors show a more significant influence than husbandry system on cecum microbial diversity of chickens when fed the same diet.

\section{CONFLICT OF INTEREST}

We certify that there is no conflict of interest with any financial organization regarding the material discussed in the manuscript.

\section{ACKNOWLEDGMENTS}

The authors thank the Project Funders: The project from the Fundamental Research Funds for the Central Universities of China (Project No. 2662013PY008) and the project of innovative actions on agricultural science and technology in Hubei province: innovation on environmental friendly feeding technologies of livestock and poultry with high quality and efficiency (Project No. 2018skjcx05) for financing this study, Ecological Chicken Farm in Jianshi County of Hubei Province for supporting the materials in this study. The authors also thank the supervisor for the guidance of the test design and the help of the partners.

\section{REFERENCES}

1. Akyurek H, Orhan ZC. Effects of phytase supplementation on the performance and egg quality of free-range layers. Int $J$ Curr Res 2016;8:44142-7.

2. Sundrum A. Organic livestock farming: a critical review. Livest Prod Sci 2001;67:207-15. https://doi.org/10.1016/ S0301-6226(00)00188-3

3. Albokhadai I. Hematological and some biochemical values of indigenous chickens in Al-Ahsa, Saudi Arabia during summer season. Asian J Poult Sci 2012;6:138-45. https://doi. org/10.3923/ajpsaj.2012.138.145

4. Danielsen M, Hornshøj H, Siggers RH, Jensen BB, van Kessel AG, Bendixen E. Effects of bacterial colonization on the porcine intestinal proteome. J Proteome Res 2007;6:2596-604. https:// doi.org/10.1021/pr070038b

5. Les D, Margaret MFN, Relman DA. An ecological and evolutionary perspective on human-microbe mutualism and disease. Nature 2007;449:811-8. https://doi.org/10.1038/ nature 06245

6. Turnbaugh P, Hamady M, Yatsunenko T, et al. A core gut microbiome in obese and lean twins. Nature 2009;457:4804. https://doi.org/10.1038/nature07540

7. Zhao L, Wang G, Siegel P, et al. Quantitative genetic background of the host influences gut microbiomes in chickens. Sci Rep 2013;3:1163. https://doi.org/10.1038/srep01163

8. Shira EB, Sklan D, Friedman A. Impaired immune responses in broiler hatchling hindgut following delayed access to feed. Vet Immunol Immunopathol 2005;105:33-45. https://doi.org/ 10.1016/j.vetimm.2004.12.011

9. Niba AT, Beal JD, Kudi AC, Brooks PH. Bacterial fermentation in the gastrointestinal tract of non-ruminants: Influence of fermented feeds and fermentable carbohydrates. Trop Anim Health Prod 2009;41:1393. https://doi.org/10.1007/s11250009-9327-6

10. Wenk C. The role of dietary fibre in the digestive physiology of the pig. Anim Feed Sci Technol 2001;90:21-33. https://doi. org/10.1016/S0377-8401(01)00194-8

11. Gong J, Yu H, Liu T, et al. Effects of zinc bacitracin, bird age and access to range on bacterial microbiota in the ileum and caeca of broiler chickens. J Appl Microbiol 2008;104:137282. https://doi.org/10.1111/j.1365-2672.2007.03699.x

12.Gong J, Forster RJ, Yu H, et al. Diversity and phylogenetic analysis of bacteria in the mucosa of chicken ceca and comparison with bacteria in the cecal lumen. FEMS Microbiol Lett 2002;208:1-7. https://doi.org/10.1111/j.1574-6968.2002. tb11051.x

13.Smirnov A, Perez R, Amit-Romach E, Sklan D, Uni Z. Mucin dynamics and microbial populations in chicken small intestine are changed by dietary probiotic and antibiotic growth promoter supplementation. J Nutr 2005;135:187-92. https:// doi.org/10.1093/jn/135.2.187 
14.Lan PTN, Hayashi H, Sakamoto M, Benno Y. Phylogenetic analysis of cecal microbiota in chicken by the use of $16 \mathrm{~S}$ rDNA clone libraries. Microbiol Immunol 2002;46:371-82. https:// doi.org/10.1111/j.1348-0421.2002.tb02709.x

15. Kozich JJ, Westcott SL, Baxter NT, Highlander SK, Schloss PD. Development of a dual-index sequencing strategy and curation pipeline for analyzing amplicon sequence data on the MiSeq Illumina sequencing platform. Appl Environ Microbiol 2013;79:5112-20. https://doi.org/10.1128/AEM. 01043-13

16. Carmody R, Gerber G, Luevano Jr JM, et al. Diet Dominates host genotype in shaping the murine gut microbiota. Cell Host Microbe 2015;17:72-84. https://doi.org/10.1016/j.chom.2014. 11.010

17.Zhao L, Wang G, Siegel P, et al. Quantitative genetic background of the host influences gut microbiomes in chickens. Sci Rep 2013;3:1163. https://doi.org/10.1038/srep01163

18. Bailey MT, Dowd SE, Parry NM, Galley JD, Schauer DB, Lyte M. Stressor exposure disrupts commensal microbial populations in the intestines and leads to increased colonization by Citrobacter rodentium. Infect Immun 2010;78:1509-19. https://doi.org/10.1128/IAI.00862-09

19. Choe DW, Loh TC, Foo HL, Hairbejo M, Awis QS. Egg production, faecal $\mathrm{pH}$ and microbial population, small intestine morphology, and plasma and yolk cholesterol in laying hens given liquid metabolites produced by Lactobacillus plantarum strains. Br Poult Sci 2012;53:106-15. https://doi.org/10.1080 /00071668.2012.659653

20.Lan PTN, Hayashi H, Sakamoto M, Benno Y. Phylogenetic analysis of cecal microbiota in chicken by the use of $16 \mathrm{~S}$ rDNA clone libraries. Microbiol Immunol 2002;46:371-82. https://doi.org/10.1111/j.1348-0421.2002.tb02709.x

21.Xu Y, Yang H, Zhang L, et al. High-throughput sequencing technology to reveal the composition and function of cecal microbiota in Dagu chicken. BMC Microbiol 2016;16:259. https://doi.org/10.1186/s12866-016-0877-2

22.Zhou X, Jiang X, Yang C, et al. Cecal microbiota of Tibetan
Chickens from five geographic regions were determined by 16S rRNA sequencing. Microbiologyopen 2016;5:753-62. https://doi.org/10.1002/mbo3.367

23.Ley RE, Micah H, Catherine L, et al. Evolution of mammals and their gut microbes. 2008;320:1647-51. https://doi.org/ 10.1126/science. 1155725

24.Leser TD, Amenuvor JZ, Jensen TK, Lindecrona RH, Mette B, Kristian ML. Culture-independent analysis of gut bacteria: the pig gastrointestinal tract microbiota revisited. Appl Environ Microbiol 2002;68:673-90. https://doi.org/10.1128/AEM.68.2. 673-690.2002

25. Turnbaugh PJ, Ley RE, Mahowald MA, Vincent M, Mardis ER, Gordon JI. An obesity-associated gut microbiome with increased capacity for energy harvest. Nature 2006;444:102731. https://doi.org/10.1038/nature05414

26. Carlotta DF, Duccio C, Monica DP, et al. Impact of diet in shaping gut microbiota revealed by a comparative study in children from Europe and rural Africa. Proc Natl Acad Sci USA 2010;107:14691-6.

27. Saengkerdsub S, Herrera P, Woodward CL, Anderson RC, Nisbet DJ, Ricke SC. Detection of methane and quantification of methanogenic archaea in faeces from young broiler chickens using real-time PCR. Lett Appl Microbiol 2007;45: 629-34. https://doi.org/10.1111/j.1472-765X.2007.02243.x

28. Lu J, Idris U, Harmon B, Hofacre C, Maurer JJ, Lee MD. Diversity and succession of the intestinal bacterial community of the maturing broiler chicken. Appl Environ Microbiol 2003; 69:6816-24. 10.1128/AEM.69.11.6816-6824.2003

29. Latham MJ, Wolin MJ. Fermentation of cellulose by Ruminococcus flavefaciens in the presence and absence of Methanobacterium ruminantium. Appl Environ Microbiol 1977;34: 297-301.

30.Sybille T, June Z, Michael K, Roy M, Marco ML. The intestinal microbiota in aged mice is modulated by dietary resistant starch and correlated with improvements in host responses. FEMS Microbiol Ecol 2013;83:299-309. https://doi.org/10. 1111/j.1574-6941.2012.01475.x 